\title{
Magnetic resonance imaging of the forefoot: A concise, practical overview
}

\author{
Author: \\ Mark Velleman ${ }^{1}$ \\ Affiliation: \\ ${ }^{1}$ Department of Radiology, \\ University of Pretoria, \\ South Africa \\ Correspondence to: \\ Mark Velleman \\ Email: \\ mdvelleman@gmail.com \\ Postal address: \\ PO Box 11315 , \\ Silver Lakes 0054, \\ South Africa \\ Dates: \\ Received: 04 Sept. 2014 \\ Accepted: 04 Nov. 2014 \\ Published: 12 Dec. 2014 \\ How to cite this article: \\ Velleman, M. Magnetic \\ resonance imaging of \\ the forefoot: A concise, \\ practical overview. S Afr J \\ Rad. 2014;18(2); Art. \#732, \\ 5 pages. http://dx.doi. \\ org/10.4102/sajr.v18i2.732

\section{Copyright:} \\ (C) 2014. The Authors. \\ Licensee: AOSIS \\ OpenJournals. This \\ work is licensed under \\ the Creative Commons \\ Attribution License.
}

Read online:
The forefoot may be affected by a wide variety of pathologies that require imaging. Magnetic resonance imaging is the modality of choice for soft-tissue pathologies and occult bone lesions. Correct imaging protocols and an awareness of anatomy are essential for accurate diagnosis.

\section{Introduction}

A sound knowledge of forefoot anatomy, a basic understanding of applicable biomechanics, and a structured differential diagnosis, are essential when performing imaging of the forefoot.

\section{Anatomy}

The foot is divided into the hind foot, mid foot and forefoot. The hind foot comprises the talus and calcaneus; the mid foot comprises the navicular, cuboid, medial, intermediate and lateral cuneiforms; and the forefoot comprises the metatarsal bones and phalanges. Magnetic resonance imaging (MRI) examinations are usually divided into the (1) ankle and hind foot; (2) mid foot; and (3) forefoot examinations. A dedicated examination technique and protocol is essential for each region.

\section{Magnetic resonance imaging technique}

High-resolution images of the forefoot should be obtained in three anatomical planes. If the region of suspected pathology is uncertain, a large field of view (FOV) short tau inversion recovery (STIR) examination of the ankle and foot $(200 \mathrm{~mm}-270 \mathrm{~mm})$ may be obtained, to identify a specific region of interest (ROI). Dedicated forefoot, mid foot or ankle examinations may then be performed. The use of dedicated foot and ankle coils is advised but, if these are not available, knee, head or flexible coils can be used as a substitute. Imaging of the feet should be obtained separately, as large FOV examinations of both the foot and ankle in one coil does not yield adequate imaging quality. ${ }^{1} \mathrm{~A}$ vitamin $\mathrm{E}$ tablet can be used as a skin marker to identify the region of discomfort (Figure 1). ${ }^{2,3}$

\section{Supine versus prone examination position}

A supine position is more comfortable for the patient, but the prone position has been advocated for cases with suspected Morton's neuroma, ${ }^{2}$ as biomechanical action in the prone position aids in visualising this condition.

\section{Imaging planes}

Imaging planes are prescribed from the scout views. ${ }^{3}$ Initially, three-axis short scout views are obtained (acquisition time 40 seconds), followed by slightly high resolution scout views in the short and long axis (acquisition time 50 seconds), after which the first sagittal diagnostic sequence is prescribed (Figure 2).

\section{Anatomical planes for forefoot imaging}

The sagittal plane is usually prescribed in the long axis of the second metatarsal, but may be adjusted according to the ROI. The short axis is prescribed in the axis of the second or third metatarsal perpendicular to the sagittal and long axis planes. The long axis is prescribed in the axis of the second or third metatarsal on sagittal views. ${ }^{2}$

It is important to avoid confusion when identifying the imaging planes. We prefer to use short and long axis terminology, rather than coronal and axial descriptions, to avoid confusion because positioning (prone versus supine) can influence the relative plane in relation to the long axis of the body. ${ }^{3}$ 


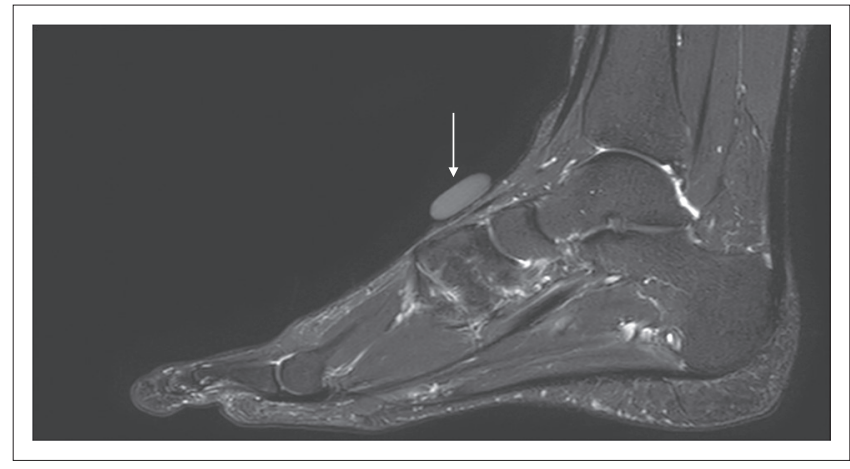

FIGURE 1: Sagittal T2 FS MR image demonstrating a vitamin E capsule (arrow) used to indicate the region of discomfort.
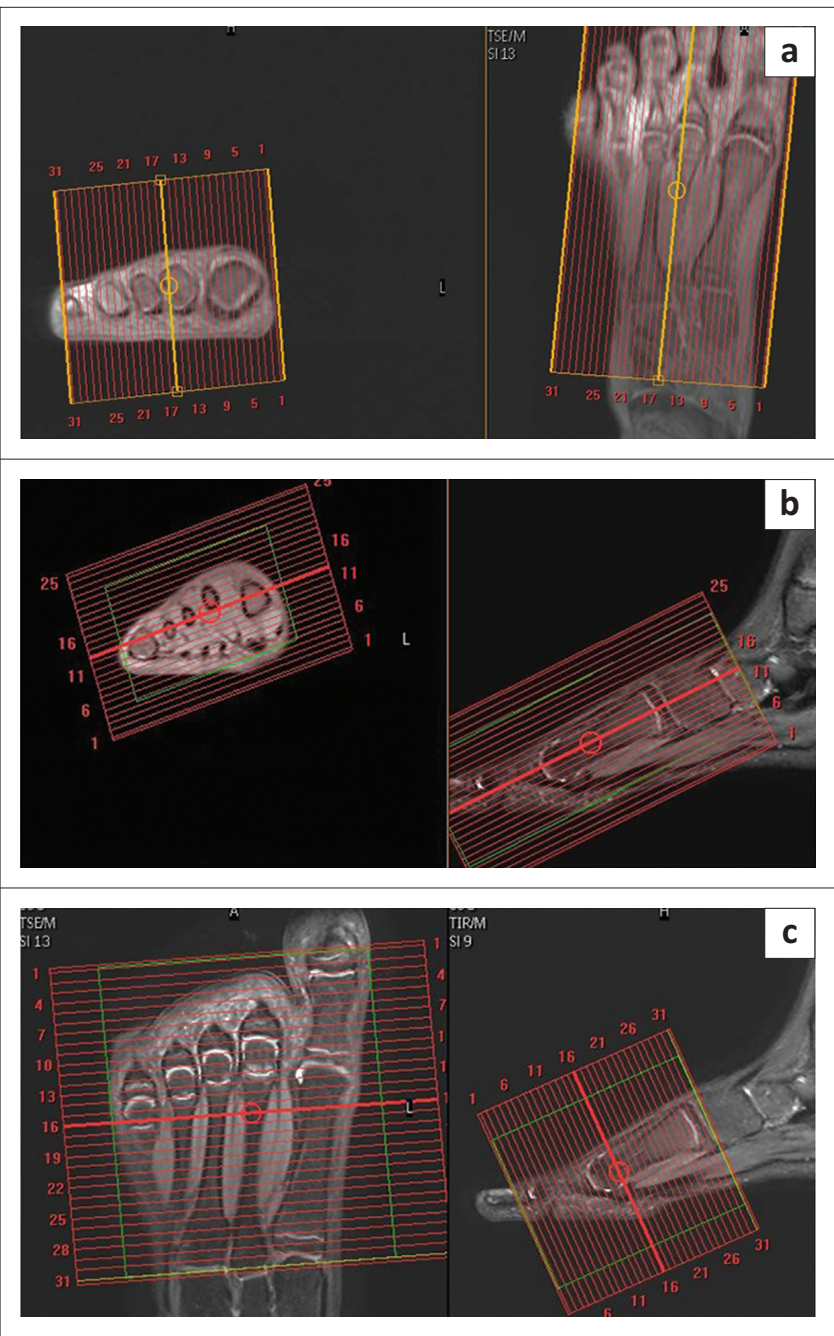

FIGURE 2: Scout views used to plan imaging planes. (a) sagittal plane, (b) long axis plane and (c) short axis plane.

\section{Sequences}

A combination of non-fat-saturated (proton density (PD)/ T1) and fluid-sensitive fat-saturated (PD FS/T2 FS) image sequences are obtained, with all sequences preferably in three imaging planes. Typical echo times for fluid-sensitive sequences are $30 \mathrm{~ms}-60 \mathrm{~ms}$ but are adjusted to vendor variations and magnetic field strength. The field of view is
$120 \mathrm{~mm}-220 \mathrm{~mm}$ and slice thickness is $2.5 \mathrm{~mm}-3 \mathrm{~mm}$, with a small slice gap $(10 \%-20 \%)$.

\section{Additional sequences}

A STIR sequence is obtained if adequate fat saturation is not obtained, or when dealing with metal or other artefacts. Gradient echo sequences are added when searching for foreign bodies, blood products or calcifications. T1 FS with or without intravenous gadolinium is reserved for special cases such as mass lesions and infections. Routine use of contrast when evaluating Morton's neuroma is debatable.

\section{MR short axis anatomy}

Compartmental anatomy is crucial when evaluating forefoot pathology and is best imaged in the short axis plane. The deep transverse metatarsal ligament divides the forefoot into the dorsal and plantar compartments. The dorsal compartment contains the metatarsals and intermetatarsal spaces including the intermetatarsal bursae. The plantar compartment contains the important neurovascular bundles, specifically important when imaging for a possible Morton's neuroma (Figure 3). The first metatarsophalangeal and sesamoid complex anatomy is also visualised in the short axis plane (Figure 4).

\section{Biomechanics}

Human bipedalism has evolved over a period of approximately 4 million years. During this process, the first tarso-metatarsal joint has not evolved into an adequately rigid joint, with the medial longitudinal arch also not sufficiently rigid to absorb continuous high-stress loads. When the medial longitudinal arch starts to sag, usually owing to old age or underlying foot pathology such as rheumatoid arthritis or other joint-centred pathology, secondary load transference occurs to the second digit and lateral arch, with also a relatively rigid second tarso-metatarsal joint.

Stress-load transference may lead to tibialis posterior dysfunction, and stress changes in the second metatarsal with secondary pressure changes in the second metatarsal head and peripheral soft-tissue pressure point or plantar plate. A short first metatarsal index (Greek/Morton's foot) also contributes to an increased risk and earlier occurrence of this cascade of events. ${ }^{4,5}$

\section{Pathology}

The following are differential diagnoses for forefoot pain: ${ }^{6}$

1. Morton's neuroma v. true neuroma (rare neurofibroma/ schwannoma) (Figure 5)

2. intermetatarsal bursitis (Figure 6)

3. stress fractures (Figure 7)

4. stress reaction

5. Freiberg's disease/metatarsal osteochondritis (Figure 8) 


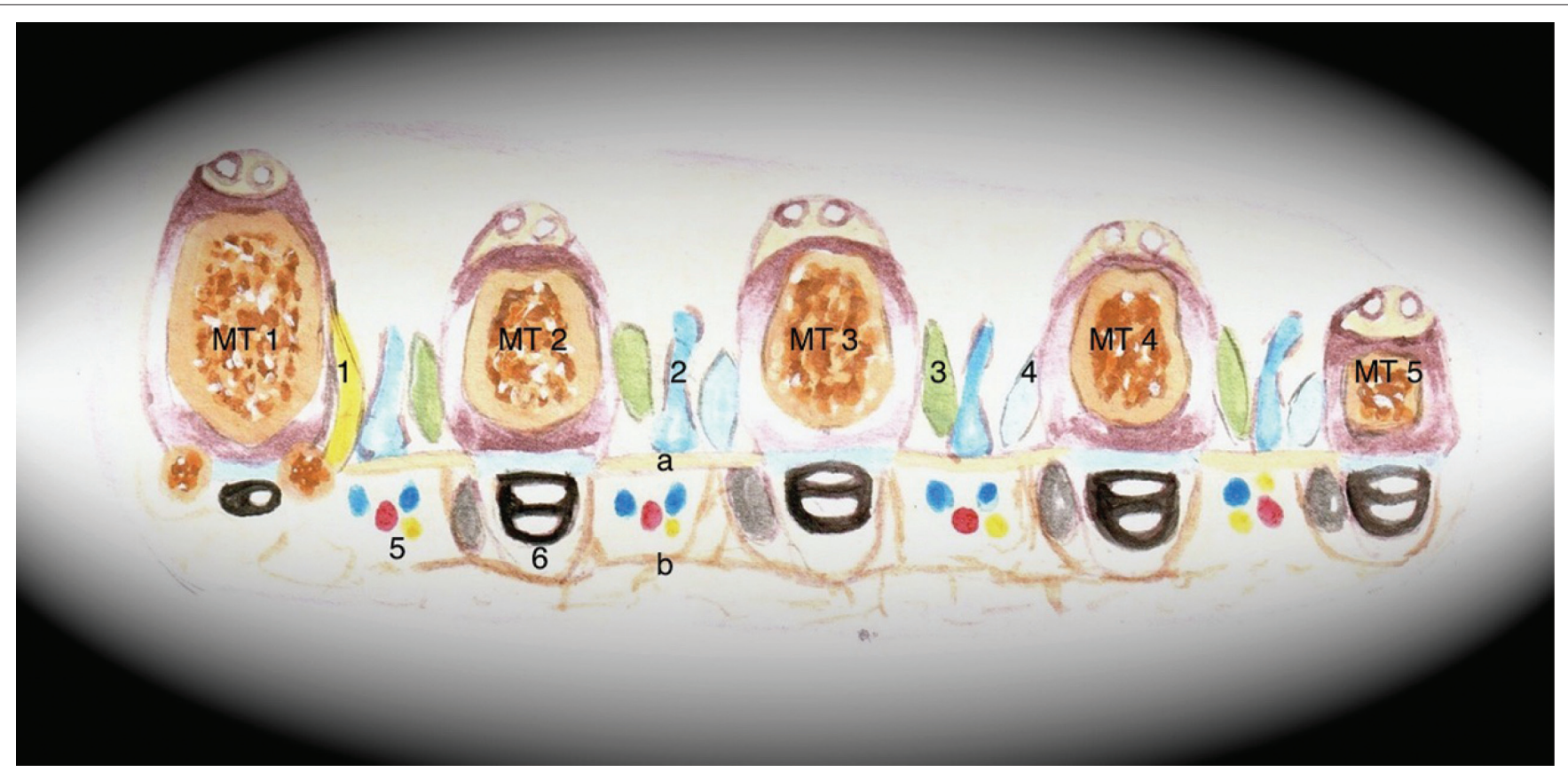

MT1-MT6, metatarsals.

1, adductor hallucis; 2 , intermetatarsal bursa; 3 , dorsal interosseous tendon; 4, plantar interosseous tendon; 5 , neurovascular bundles; 6, flexor digitorum tendons. a, deep transverse metatarsal ligament; b, superficial transverse metatarsal ligament.

FIGURE 3: Schematic diagram of forefoot anatomy in the short axis view.

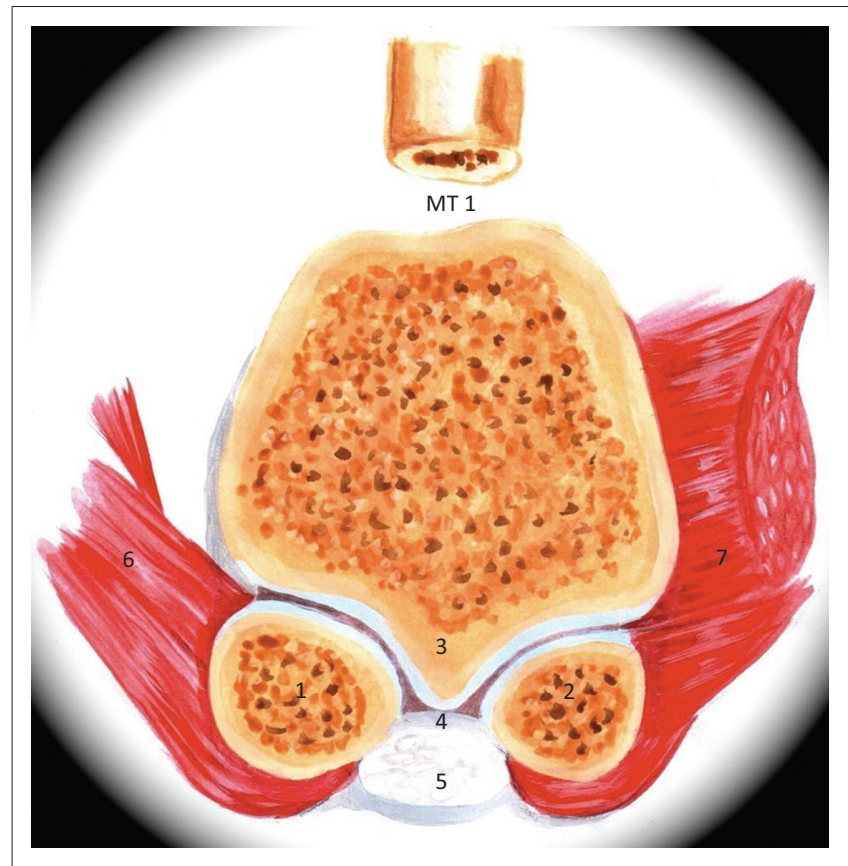

MT1, 1st metatarsal; 1, medial sesamoid; 2, lateral sesamoid; 3, crista; 4, intersesamoidal liga ment; 5 , flexor hallucis longus; 6 , abductor hallucis; 7 , adductor hallucis.

FIGURE 4: Schematic diagram of the anatomy of the first metatarsophalangeal sesamoid complex in the short axis view.

6. sesamoiditis/fracture/osteonecrosis (Figure 9)

7. rheumatoid nodules (Figure 10)

8. giant cell tumour of the tendon sheath (Figure 11)

9. miscellaneous: synovial cyst, chondroma of soft tissue, foreign-body granuloma, plantar fibromatosis, bony lesions (Figure 12)

10. trauma to bone and soft tissue.
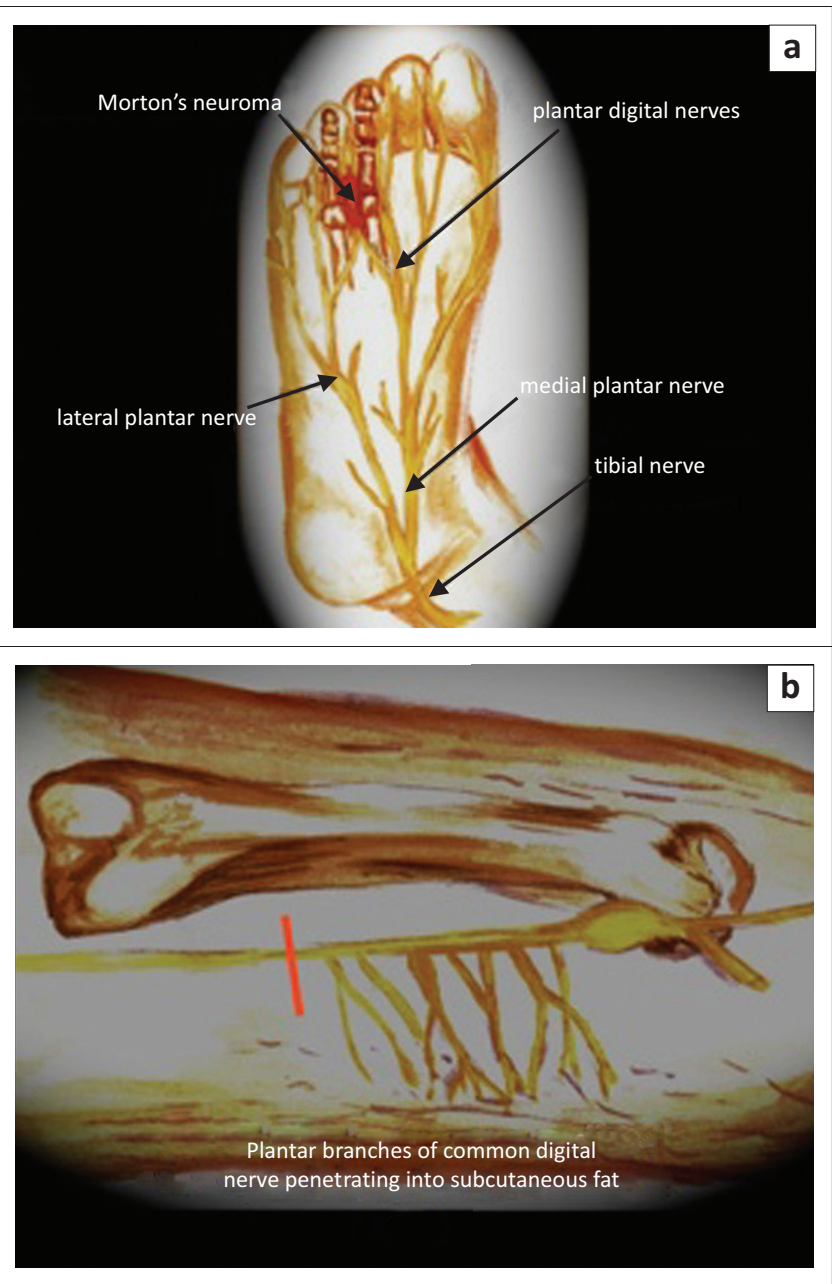

FIGURE 5: Schematic diagram illustrating the location of (a) Morton's neuroma occurring in the webspace between the 3rd and 4th toes; and (b) the region of peripheral nerve where excision should occur to prevent the formation of a stump neuroma. 


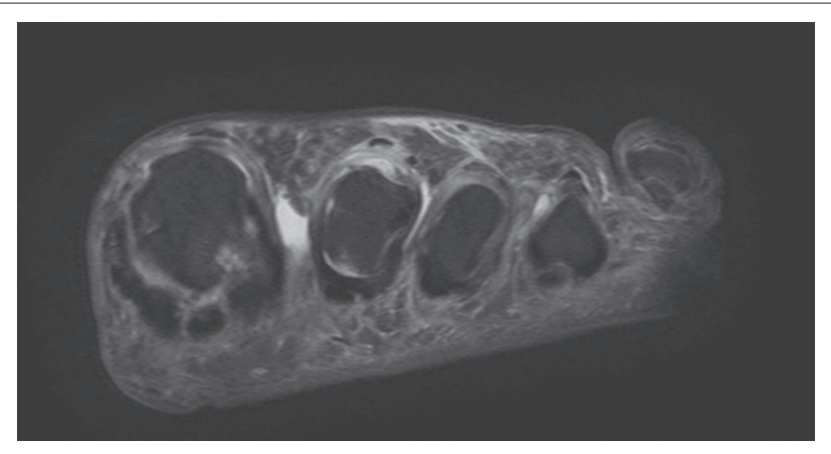

FIGURE 6: Short axis T2 FS MR image of the foot demonstrating an intermetatarsa bursitis.

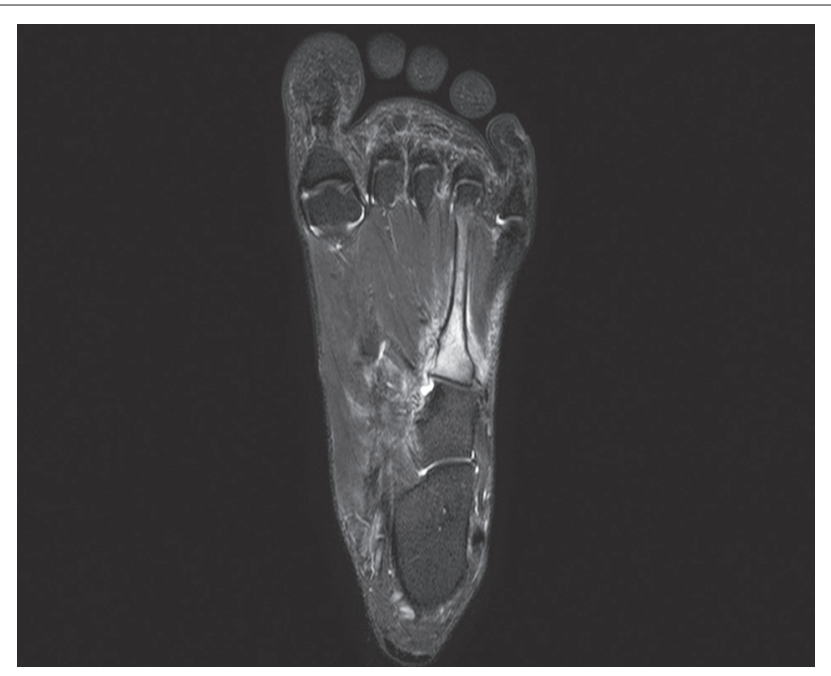

FIGURE 7: Long axis STIR MR image of the foot demonstrating oedema in the 4th metatarsal owing to a stress fracture.

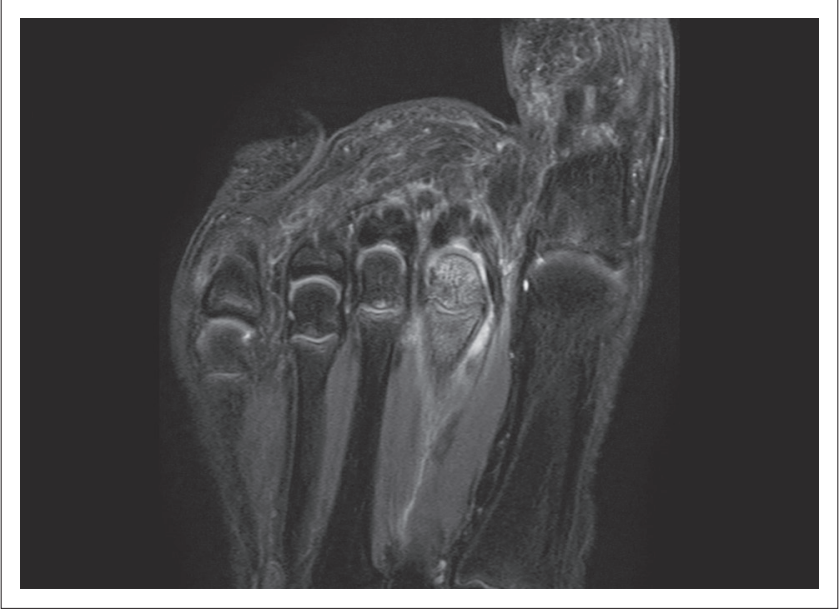

FIGURE 8: Long axis T2 FS MR image of the foot demonstrating oedema in the 2nd metatarsal head owing to Freiberg's disease.

\section{Teaching points}

1. High-resolution true plane anatomical imaging is important for accurately diagnosing forefoot pathology and abnormalities.

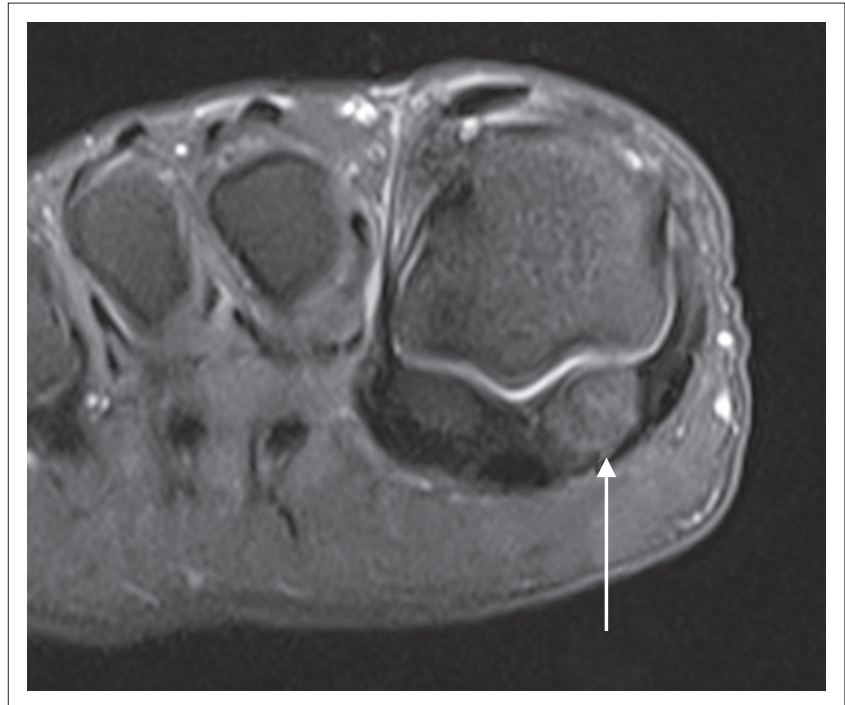

FIGURE 9: Short axis T2 FS MR image of the foot demonstrating oedema in the medial sesamoid (arrow) owing to sesamoid stress changes.

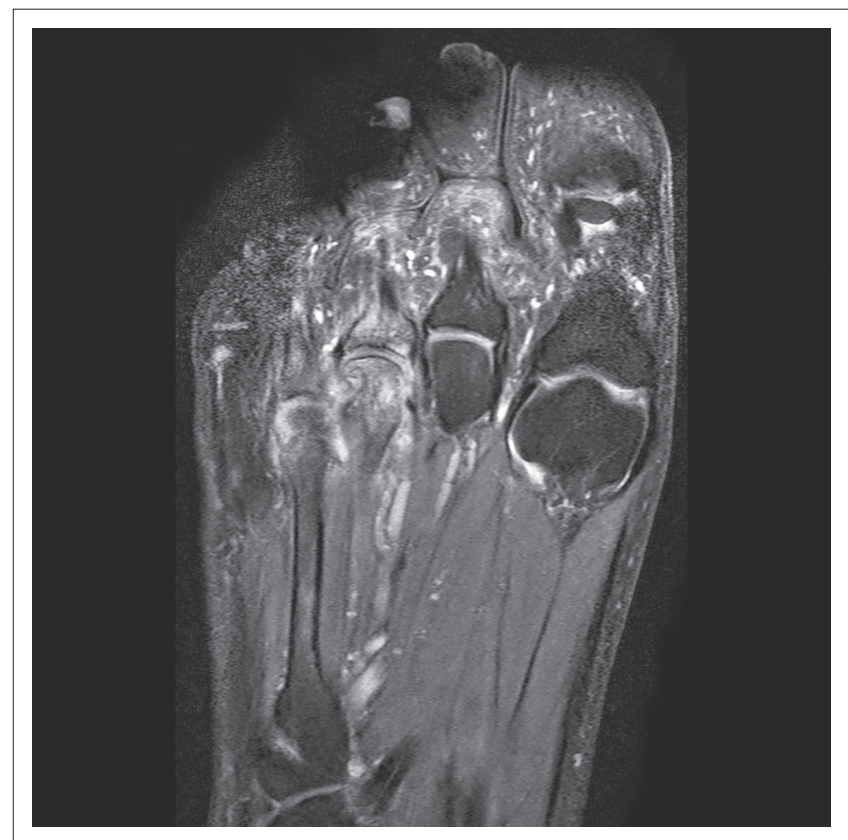

FIGURE 10: Long axis T2 FS MR image of the foot demonstrating synovitis, erosions and bone oedema in a patient with rheumatoid arthritis.

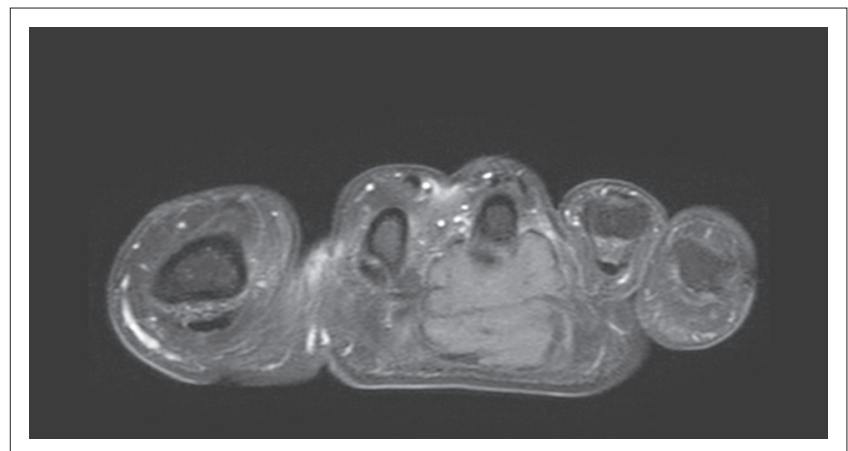

FIGURE 11: Short axis T1 FS MR image of the foot demonstrating a giant cell tumour in the tendon sheath of the 3rd toe. 


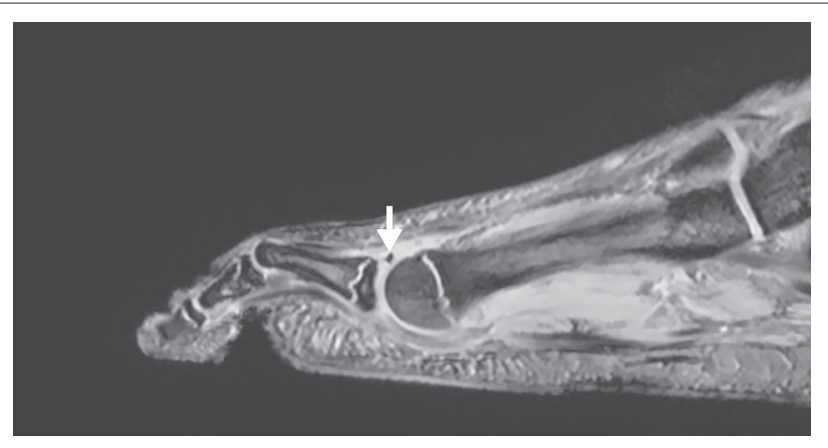

FIGURE 12: Sagittal gradient recalled echo (GRE) MR image of the foo demonstrating a foreign body (arrow) in the plantar aspect of the foot.

2. A basic understanding of forefoot biomechanics is valuable when assessing forefoot pain.

3. A sound knowledge of the differential diagnoses of forefoot pathology, as well as a list of the most likely causes of forefoot pain, is important.

\section{Conclusion}

A sound knowledge of normal anatomy, biomechanics, differential pathology and the most common causes of forefoot pain is essential when imaging the forefoot. High-resolution anatomical imaging in three planes should be obtained with sequences specifically tailored for the forefoot. Fat-saturated fluid-sensitive as well as nonfat-saturated PD or T1 sequences should be included. In specific instances, STIR, gradient echo or post-contrast image sequences may be added. If there is uncertainty about the specific region of pathology, a large FOV STIR study may be obtained to identify the region of pathology.

\section{Acknowledgements}

I thank Dr Marinel Gericke for her assistance with the illustrations.

\section{Competing interests}

The author declares that they he has no financial or personal relationships that he might have inappropriately influenced him in writing this article.

\section{References}

1. Erickson SJ, Rosengarten JL. MR imaging of the forefoot: Normal anatomical findings. AJR 1993;160(3):565-571. http://dx.doi.org/10.2214/ ajr.160.3.8430554

2. Needell S. Guide to MR imaging of the foot \& ankle. Boca Radiology Group [homepage on the Internet]. [cited January 2014]. Available from: http://www. bocaradiology.com/foot/index.html

3. Arnold G, Saifuddin V, Marcantonio D, Shashin D. Normal magnetic resonance imaging anatomy of the ankle and foot. Magn Reson Imaging Clin N Am. 2011;19(3):655-679. http://dx.doi.org/10.1016/j.mric.2011.05.010

4. Rothbart BA. An explanation of Morton's foot aka Morton's toe and Greek foot. Curing chronic pain. [homepage on the Internet]. [cited January 2014]. Available from: http://curingchronicpain.com/an-explanation-of-mortons-foot-aka-mortonstoe-and-greek-foot

5. Wikipedia. Morton's toe. [homepage on the Internet]. [cited January 2014] Available from: http://en.wikipedia.org/wiki/Morton's_toe

6. Zanetti M, Weishaupt D. MR imaging of the forefoot: Morton neuroma and differential diagnosis. Semin Musculoskelet Radiol. 2005;9(3):175-186. http:// dx.doi.org/10.1055/s-2005-921938 